\title{
High temperature indentation behavior of eutectic lead-free solder materials
}

\author{
W.H. Müller ${ }^{\mathrm{a}}$ and H. Worrack
}

Technische Universität Berlin, Fakultät V, Verkehrs- und Maschinensysteme, Institut für Mechanik, LKM, Sekr. MS 2, Einsteinufer 5, 10587, Berlin, Germany

\begin{abstract}
Electronic malfunction caused by thermal stresses is one major problem in modern electronic industries. Therefore, the precise knowledge of the mechanical solder material properties as a function of temperature is required. Nanoindentation and its potential of recording load-displacement curves is a widely-used miniature test for the determination of Young's modulus and hardness values. Furthermore, such tests can be performed in a temperature range from Room Temperature (RT) up to $+500^{\circ} \mathrm{C}$ by using a Hot-Stage add on. In this paper the lead-free solder alloys Sn91Zn9 and Sn42Bi58, and also copper and fused silica, which is used for the indenter calibration are investigated. The results for quartz and copper agree with the published values in several references. However, the Young's modulus of Sn42Bi58 as a function of temperature differs from the values presented in the literature. Due to delayed material response in the unloading regime it must be assumed that creep effects lead to an incorrect automatic data evaluation. Investigation and understanding of the creep behavior is part of this paper. For this purpose a visco-elastic material model is used to model the indentation response at elevated temperatures and to determine the corresponding viscous material constants.
\end{abstract}

\section{Introduction}

The determination of mechanical solder material properties provides a quantitative basis for reliable new product developments in microelectronics. In context with the requirement of finding low-cost lead-free solders, material tests were performed for Sn91Zn9 and Sn42Bi58.

The nanoindentation system NanoTest from Micro Materials Ltd. is an important tool to determine the local mechanical properties of very small structures, e.g., microelectronic solder joints. The procedure is comparable to the well known Vickers hardness measurements. The main difference consists in using a triangular pyramid indenter (Berkovich tip) instead of a square-based pyramid. Moreover nanoindentation tests are performed using very small loads $(\mathrm{mN})$ resulting in very small indents on the specimen surface $(\mu \mathrm{m})$.

During the indentation process force and indentation depth are recorded and depicted in a characteristic load $v s$. indentation-depth curve. According to Oliver and Pharr the recorded data allows obtaining hardness and Young's modulus values directly from the measurement.

\footnotetext{
a e-mail : wolfgang.h.mueller@tu-berlin.de
}

This is an Open Access article distributed under the terms of the Creative Commons Attribution-Noncommercial License 3.0, which permits unrestricted use, distribution, and reproduction in any noncommercial medium, provided the original work is properly cited. 


\section{Nanoindentation}

\subsection{Nanoindentation setup}

The setup of the nanoindentation system (including the hot stage add-on) is presented in Figs. 1/2.
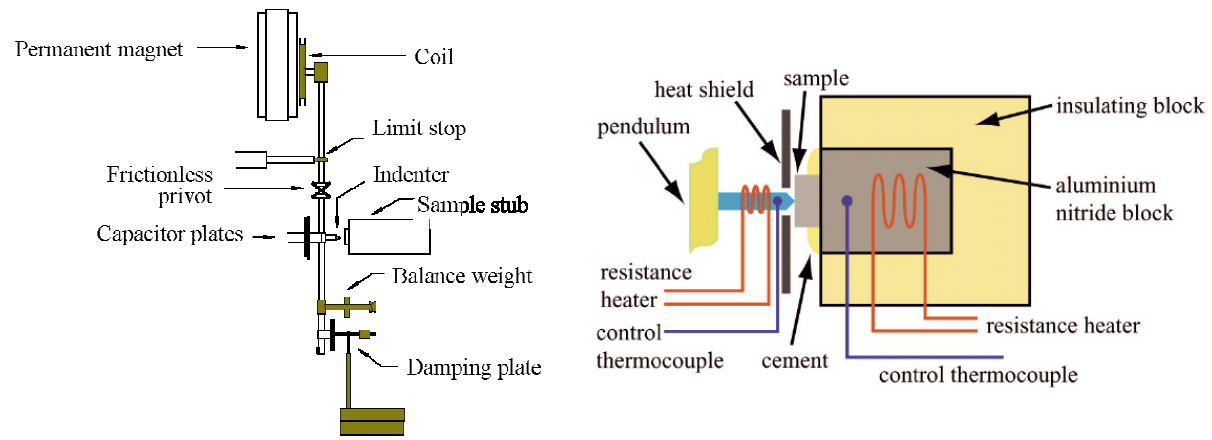

Fig. 1. Schematic of the nanoindenter (left) and of the hot stage (right).
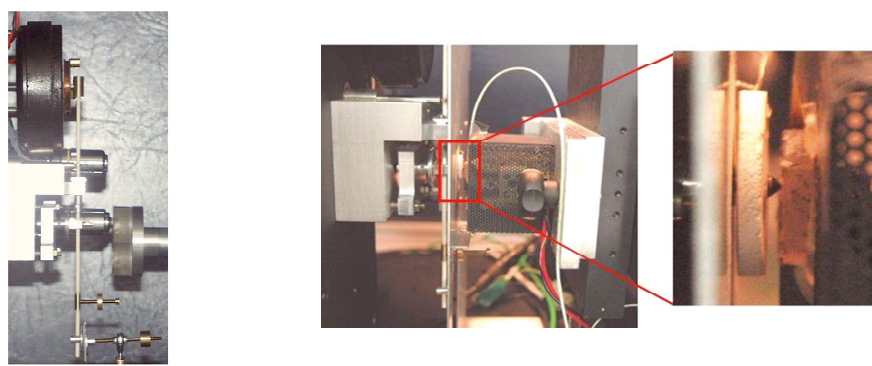

Fig. 2. Photograph of the nanoindenter (left) and of the hot stage (right).

The Berkovich indenter, fixed on a pendulum, is aligned horizontally. A permanent magnet, placed at the upper end of the pendulum, generates a magnetic force and moves the pendulum tip to the left. Correspondingly, the pendulum moves counterclockwise about the center of rotation. As a result the indenter tip moves to the right and indents the material on the specimen holder. The movement of the indenter, and thus the indentation depth, is measured by a Wheatstone bridge. Settings and calibration of the system are operated from an external computer to shield the system from the outside which might influence the accuracy of the measurement. An additional chamber surrounds the system and protects the setup from further external disturbances.

Investigations of the influence of temperature on the mechanical material properties require a hot stage: An aluminum nitride block is heated by a resistance heater up to the target temperature assigned by the computer. The control thermocouple, next to the heater, measures the current temperature and is used by the computer for temperature control. The diamond indenter is heated as well to avoid temperature gradients between the indenter and the sample surface. A second thermocouple is required to measure the current temperature on the sample surface. This temperature is used to relate the possible change in hardness and Young's modulus to temperatures. In addition a heat shield located between the sample surface and the pendulum protects the nanoindenter setup and the measurement equipment.

\subsection{Temperature measurements}

It was indicated that a potential difference in temperature between the control thermocouple and the thermocouple on the sample surface has to be monitored. Tests were first performed with the 
calibration material fused silica and with copper (Cu-HCP), for which some information on the temperature dependence of Young's modulus was available. The abbreviation HCP stands for High Conductivity Phosphorus deoxidized copper. The specimens were fixed on the sample holder and the current temperature on the sample surface was recorded, $c f$., Fig 3. Target temperatures were assigned between $50^{\circ} \mathrm{C}$ and $500^{\circ} \mathrm{C}$. Fig. 4 shows the deviation of temperature in percent.

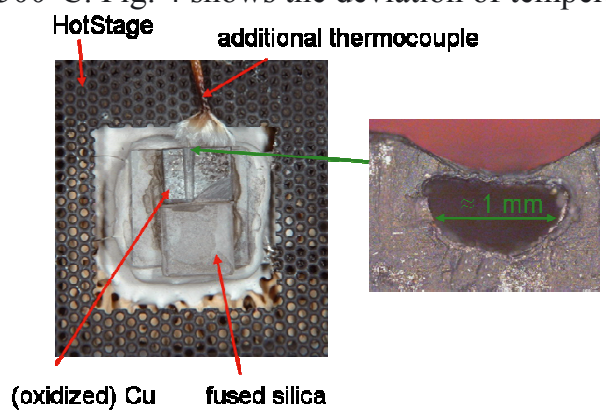

Fig. 3. Top view of the indentation area.
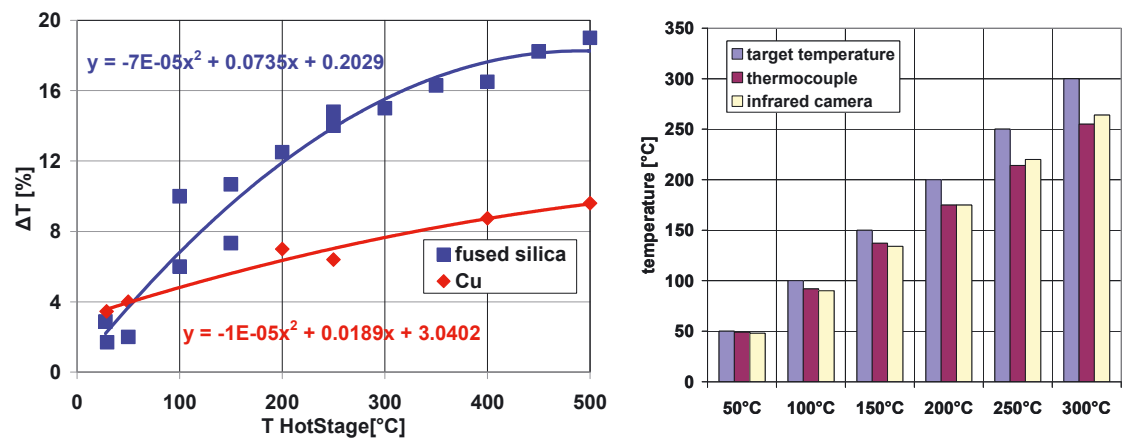

Fig. 4. Temperature differences between target and measurement temperatures.

The main results of the temperature tests are, first, that the higher the target value assigned by the computer, the higher the temperature difference on the sample surface and, second, the temperature difference is material dependent, which could be attributed to the different thermal (storage) properties of the materials.

An important conclusion of these investigations is the need for additional temperature measurements during our studies. In order to check the accuracy of the thermocouple measurements the sample temperatures were examined by an infrared camera as well. The results of the infrared camera depend on the emissivity input o be provided by the user, which is difficult to asses, in particular when the texture of the specimen surface changes during the measurements, e.g., by oxidation. At a predefined temperature four measurements were performed with emissivities 0.65 , $0.80,0.90$ and 0.95 . In [1] the emissivity of fused silica is quoted as 0.93 , however, the infrared camera can only vary at intervals of 0.05 . For this reason the corresponding surface temperatures of fused silica are now determined by interpolation as presented in Fig. 4. As one can see there is only a small deviation in the temperatures between the thermocouple and the infrared camera. Due to the compactness of the nanoindentation system the temperature in our further studies was measured exclusively with thermocouples.

The sensitivity of the nanoindentation experiments, which is caused by the small loads and small indents, requires a calibration procedure before every measurement. Consequently, a depth calibration for fused silica is performed at the current temperature as explained in [2]. This calibration is necessary to relate the distance moved by the indenter during the measurement to a change in voltage of the capacitor plates ( $c f$., Fig. 1). At the beginning of the calibration the specimen, fixed on the specimen holder, is moved by a spindle drive up to the moment where the 
indenter and the specimen are just in contact. At this point the capacitor plates measure an initial voltage. Then the spindle drive moves the sample until the incoming signal of the plate capacitor is $1 / 4$ of the initial voltage value at the starting point. Now the movement of the spindle drive is put in reverse and the voltage grows. Between $1 / 3$ and $2 / 3$ of the initial value of the voltage the difference in voltage is related to the known distance moved by the sample holder. The traverse path is determined from the known treat pitch of the spindle drive. Due to the thermal expansion of the sample and the indenter materials the distance of the capacitor plates varies. A new calibration routine is required before measuring at elevated temperatures. After this procedure the actual indentation test starts with 20 measurements and indentation depths between 500-1500 nm in order to obtain a sufficient number of data points, so that statistical errors are of little consequence.

In our studies four different materials were tested. Their mechanical material properties were determined at elevated temperatures and compared to the literature. An overview is given in Table 1. In addition to the materials the indentation temperatures are listed.

Table 1. Tested materials and indentation temperatures $\left[{ }^{\circ} \mathrm{C}\right]$.

\begin{tabular}{|c|c|c|c|c|}
\hline Target Value & Fused Silica & Cu HCP & Sn91Zn9 & Sn58Bi42 \\
\hline 30 & 29 & 29 & 29.5 & 29.5 \\
\hline 70 & - & - & 68 & 68 \\
\hline 110 & - & - & 106 & 106 \\
\hline 135 & - & - & 130 & 130 \\
\hline 230 & 201 & 215 & - & - \\
\hline 430 & 361 & 397 & - & - \\
\hline
\end{tabular}

\subsection{Data evaluation}

It was mentioned above that Vickers hardness is defined as the quotient of the maximum load by the imprint area of the diamond on the sample surface. Thus a Vickers indentation experiment typically results in only one value, namely the Vickers hardness, $H V$. In contrast to this, the whole loading process is recorded during nanoindentation which leads to a characteristic load $v s$. indentation depth curve (Fig. 5). According to the equations presented in [3-5] nanohardness, $H N$, is defined as the quotient of the maximum load, $P$, over the projected area, $A_{\text {proj }}$ :

$$
H N=\frac{P}{A_{\text {proj }}} .
$$

The recorded loading curve can be separated into three parts: loading, dwell time, and unloading. The unloading part determines Young's modulus through its gradient:

$$
S=\frac{\mathrm{d} P}{\mathrm{~d} h},
$$

which is used as an intermediate quantity for calculating the reduced Young's modulus, $E_{\mathrm{r}}$, of the investigated sample material: 


$$
E_{\mathrm{r}}=\frac{\sqrt{\pi}}{2} \frac{S}{\sqrt{A}} .
$$

Now Young's modulus, $E$, of the sample material can be obtained as follows:

$$
\frac{1}{E_{\mathrm{r}}}=\frac{\left(1-v^{2}\right)}{E}+\frac{\left(1-v_{\mathrm{i}}^{2}\right)}{E_{\mathrm{i}}} \text {. }
$$

provided Young's modulus of the indenter material and both Poisson's ratios are known. Note that $E_{\mathrm{i}}=1141 \mathrm{GPa}$ and $v_{\mathrm{i}}=0.07 . v$ hardly varies and is often assumed to be roughly 0.4 for solders.

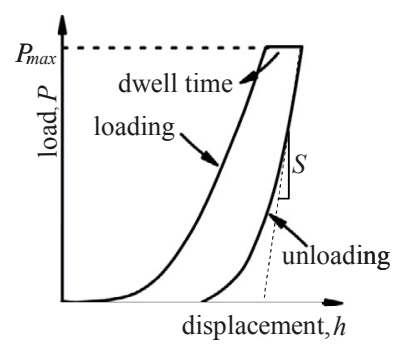

Fig. 5. Typical load indentation curve.

Furthermore, nanohardness, $H N$, together with the effective cone angle, $\gamma$, of the Berkovich indenter can be used for an estimate of the yield strength, $\sigma_{\mathrm{y}}$.

$$
\sigma_{\mathrm{y}} \approx \frac{\sqrt{3}}{2(1+\gamma)} H N .
$$

The detailed description of the data analysis and the determination of mechanical material properties from nanoindentation tests are published in a former paper by the authors [6].

\subsection{Results}
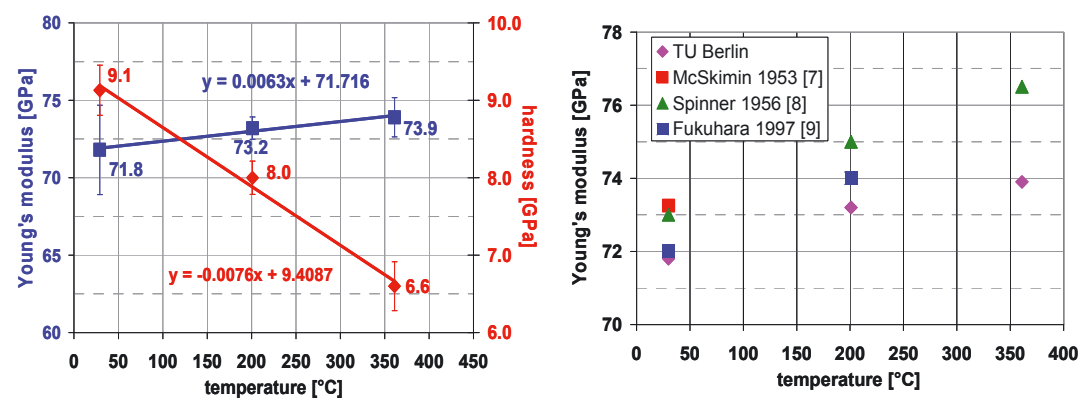

Fig. 6. $E$ and $H N$ vs. temperature (left) and comparison to literature (right) for fused silica.

Fig. 6 (left) shows the results for Young's modulus, $E$, and nanohardness, $H N$, as a function of the temperature for the calibration material, i.e., fused silica. In addition equations of the material properties as functions of temperature (in ${ }^{\circ} \mathrm{C}$ ), resulting from linear regression lines, are also presented. The somewhat unusual behavior of fused silica according to which Young's modulus increases with temperature was reported in several references [7-9]. These results are compared to 
our data in Fig. 6 (right). All references report a slight increase in a temperature range between RT and nearly $370^{\circ} \mathrm{C}$. Our values are consistent with these results and show the reliability of the measurement procedure, at least if the materials are probed at temperatures that are still well below their melting temperature. This caveat should be kept in mind later when the low-melting-pointsolders are investigated. The consistence between our indentation results in comparison to external data is shown in Fig. 7 for copper specimens as well.
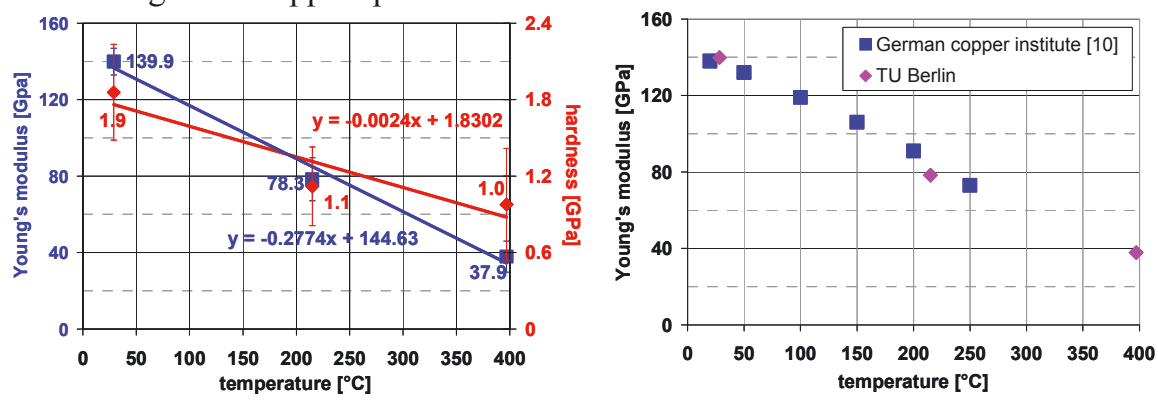

Fig. 7. $E$ and $H N$ vs. temperature (left) and comparison to literature (right) for copper.

Our investigations on lead-free Sn-solder alloys result in important information regarding their temperature-dependent mechanical properties (stiffness and yield behavior), cf. Fig. 8 .
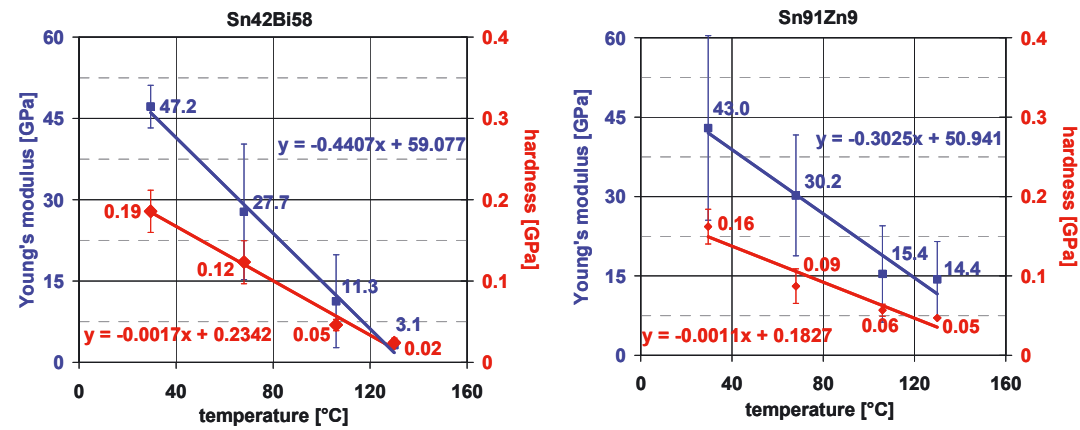

Fig. 8. $E$ and $H N \quad v s$. temperature for $\operatorname{Sn} 42 \mathrm{Bi} 58$ (left) and $\operatorname{Sn} 91 \mathrm{Zn} 9$ (right).
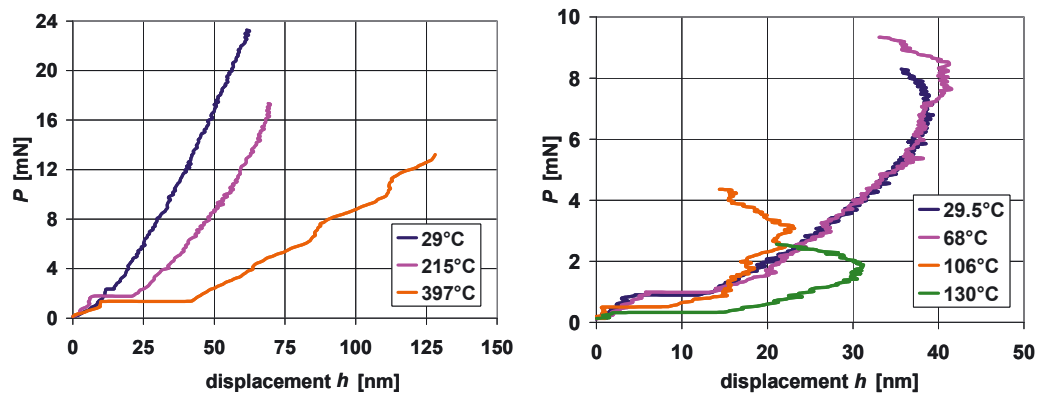

Fig. 9. Unloading curves for copper (left) and Sn42Bi58 (right).

\section{A visco-elastic model}

Creep of solders complicates the correct determination of hardness and related material properties (cf., the hold and unloading parts shown in Fig. 9, right). For this reason our future research will focus on the modeling of the "non-linear" indentation behavior by means of material models that 
take viscosity effects into account. One of the simplest rheological models is the one depicted in Fig. 10, the visco-elastic Kelvin body, which consists of two linear elastic springs (Young's moduli $E$ and $E_{1}$ ) and a linear dashpot (viscosity $\eta$ ). Stress and strain are connected as follows:

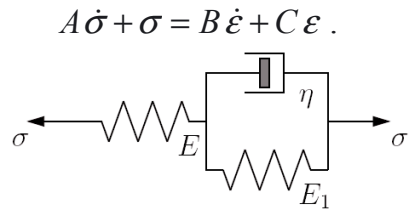

Fig. 10. Rheological model for visco-elasticity.

In this context $A, B$ and $C$ describe material dependent constants and they represent the material properties of interest $\left(A=\frac{\eta}{E+E_{1}}, B=\frac{\eta E}{E+E_{1}}, C=\frac{E E_{1}}{E+E_{1}}\right)$. Fig. 11 shows the recorded force $v s$. time (left) and displacement $v$ s. time (right) data from a nanoindentation experiment for the $\mathrm{SnBi}$ solder at RT ( $c f$. , Fig. 9, right):
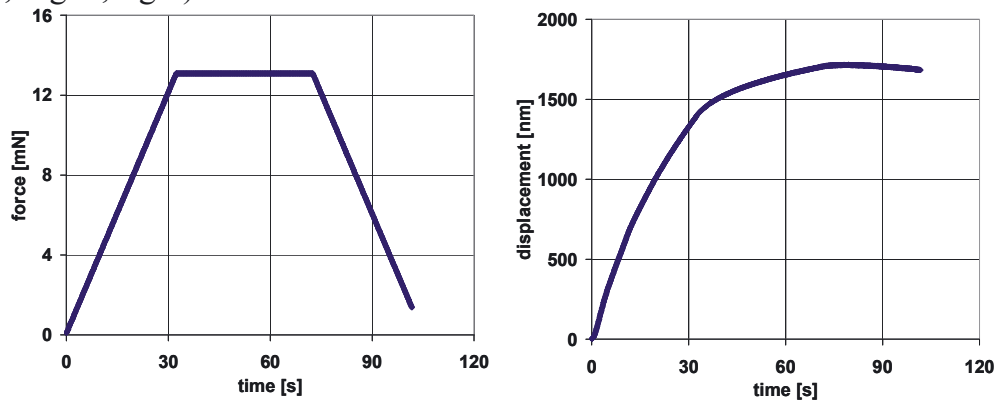

Fig. 11. Indentation raw data: force vs. time (left) and displacement vs. time (right).

Now, for starting values of $E, E_{1}$, and $\eta$ Eqn. (6) will be solved iteratively until the optimum parameters are found so that the measured displacement $v s$. time curve of Fig. 11 (right) is sufficiently reproduced. An example of this procedure is shown for dummy parameters in Fig. 12 .
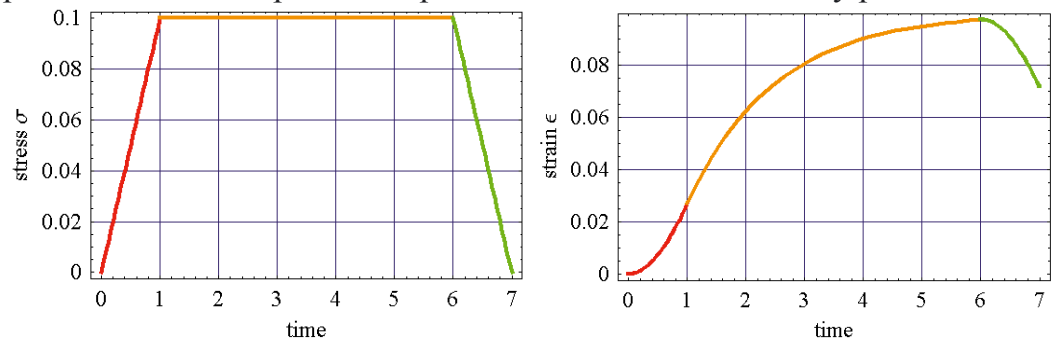

Fig. 12. Fake stress profile (left) and predicted strain vs. time curve (right).

\section{Conclusions}

The determination of local material properties requires appropriate miniature tests. Nanoindentation is able to measure load-displacement-behavior of very small structures and is an important tool for material characterization. Moreover, the temperature dependence of the material properties is of particular interest. In order to analyze the impact of temperature on Young's modulus, hardness, and yield stress nanoindentation tests at elevated temperatures were performed for fused silica, copperHCP, Sn42Bi58 and Sn91Zn9. Results for fused silica show a slight continuous increase of Young's 
modulus with temperature in contrast to a decrease in hardness. The change in Young's modulus and hardness is about $3 \%$ and $27 \%$, respectively, within a temperature range of $340^{\circ} \mathrm{C}$. The unusual behavior of the elastic modulus vs. temperature curve is compared to several references. A remarkable decrease of the values for temperature-dependent Young's modulus was detected for copper, where $E$ decreases by circa $62 \%$ for a temperature rise of $370^{\circ} \mathrm{C}$. A similar characteristic is visible in the results for eutectic Sn-solder alloys. Young's modulus of SnBi solder decreases by nearly $93 \%$ from $45 \mathrm{GPa}$ to $3.1 \mathrm{GPa}$ between $29.5^{\circ} \mathrm{C}$ and $130^{\circ} \mathrm{C}$. SnZn solder shows a slight decrease in the same temperature range. The value for Young's modulus decreases from $43 \mathrm{GPa}$ to 14.4 GPa. Our measurements result in a linearly decreasing curve to nearly zero in the investigated temperature range. A possible reason for this unexpected behavior is creep effects. At $29.5^{\circ} \mathrm{C}$ the homologous temperature of the $\mathrm{SnBi}$ solder is already 0.76 and increases to 0.98 at $130^{\circ} \mathrm{C}$. Due to this fact the beginning of the unloading part in the force $v$ s. indentation depth curves for SnBi (Fig. 9, right) shows discrepancies with the almost ideal unloading behavior of copper shown in Fig 9 (left). A comparison between the curves at $29.5^{\circ} \mathrm{C}$ and $68^{\circ} \mathrm{C}$ shows a considerable difference during the beginning of the unloading part at the maximum loads. Most likely the automated analysis of the nanoindentation software is unable to determine the correct gradient $S$ of the unloading part. Note that the $(P, h)$-curves are "nose-shaped" at the beginning of the unloading process. However, during later, the curves show a similar gradient which should lead to an almost constant Young's modulus at both temperatures, as referenced in the literature. A manual data analysis will be necessary. Due to the high melting points of fused silica and copper creep effects are not important in the investigated temperature ranges. Here our results agree with those from the mentioned references.

Despite of all this, our nanoindentation results show the importance of determining material properties as function of temperature in order to ensure the reliability of microelectronic devices. In the future the temperature dependence of several solder materials will be investigated. For this purpose creep tests and miniature tensile tests under thermal load will be performed and analyzed to investigate time-dependent plastic deformation and to characterize the solder materials even more.

\section{References}

1. VDI Wärmeatlas, $10^{\text {th }}$ revised edition, Springer, (2006)

2. Micro Materials Nano Test User Manual, Micro Materials Ltd., (2004)

3. G.M. Pharr, W.C. Oliver, Measurement of hardness and elastic modulus by instrumented indentation: Advances in understanding and refinement to methodology, Journal of Materials Research, Vol. 19, 1, pp. 3-20, (2004)

4. G.M. Pharr, W.C. Oliver, An improved technique for determining hardness and elastic modulus using load and displacement sensing indentation experiments, Journal of Materials Research, Vol. 7, 6, pp. 1564-1583, (1992)

5. A. Fischer-Cripps, Nanoindentation, Mechanical Engineering Series, Springer, New York, (2002)

6. W.H. Müller, H. Worrack, J. Sterthaus, J. Villain, J. Wilden, A. Juritza, How to extract continuum materials properties for (lead-free) solders from tensile tests and nanoindentation experiments, Microsystem Technologies, Springer, Berlin/Heidelberg, Vol. 15, 1, pp. 45-55, (2009)

7. H.J. McSkimin, Measurements of elastic constants at low temperatures by means of ultrasonic waves-data for silicon and germanium single crystals, and for fused silica, Journal of Applied Physics, Vol. 24, 8, pp. 988-997, (1953)

8. S. Spinner, Elastic moduli of glasses at elevated temperatures by a dynamic method, Journal of the American Ceramic Society, Vol. 39, 3, pp. 113-118, (1956)

9. M. Fukuhara, A. Sanpei, K. Shibuki, Low temperature elastic moduli, Debye temperature and internal dilatational and shear frictions of fused quartz, Journal of Materials Science, Vol. 32, 5, pp. 1207-1211, (1997)

10. Deutsches Kupferinstitut, $\mathrm{Cu}-\mathrm{HCP}$ data sheet from www.kupfer-institut.de 